\title{
Orientation specificity in biological motion perception
}

\author{
MARINA PAVLOVA and ALEXANDER SOKOLOV \\ University of Tübingen, Tübingen, Germany \\ and Institute of Psychology, Russian Academy of Sciences, Moscow, Russia
}

\begin{abstract}
We addressed the issue of how display orientation affects the perception of biological motion. In Experiment 1 , spontaneous recognition of a point-light walker improved abruptly with image-plane display rotation from inverted to upright orientation. Within a range of orientations from $180^{\circ}$ to $90^{\circ}$, it was dramatically impeded. Using ROC analysis, we showed (Experiments 2 and 3) that despite prior familiarization with a point-light figure at all orientations, its detectability within a mask decreased with a change in orientation from upright to a range of $90^{\circ}-180^{\circ}$. In Experiment 4, a priming effect in biological motion was observed only if a prime corresponded to a range of deviations from upright orientation within which the display was spontaneously recognizable. The findings indicate that display orientation nonmonotonically affects the perception of biological motion. Moreover, top-down influence on the perception of biological motion is limited by display orientation.
\end{abstract}

The ability to correctly identify an event is of great ecological importance for an organism, because it supports functional behavior in a continuously changing environment. Since the classic work of Gestalt psychology, there has been phenomenological evidence for veridical perception of simple events depicted only by rigid motions of several points. As will be shown later, the visual system is highly sensitive to information about the invariant structure in complex everyday events, such as biological motion patterns that consist of a set of moving dots on the main joints of an invisible walker.

Despite the potential perceptual ambiguity, humans readily extract the invariant structure from biological motion. In his initial study, Gunnar Johansson (1973, 1976 ) showed that adults need only $0.1-0.2 \mathrm{sec}$ to identify displays with filmed biological motion. Mather and West (1993) extended these findings to perception of animated point-light figures of quadrupeds. Preschoolers 3-4 years of age were able to recognize point-light hu-

Parts of the study were presented at the 12th-14th Annual Meetings of the International Society for Psychophysics and at the 19th-21st European Conferences on Visual Perception. M.P. was supported by the Deutsche Forschungsgemeinschaft (436RUS17/14/97) and by the MaxPlanck-Gesellschaft. Parts of the paper were done at the Institut für Arbeitsphysiologie, Dortmund, the Institut für Psychologie, ChristianAlbrechts-Universität zu Kiel, and the MPI for Biological Cybernetics, Tübingen. We thank Niels Birbaumer, Isabelle and Heinrich Bülthoff, C. Richard Cavonius, Walter H. Ehrenstein, Dieter Heyer, Laurence Maloney, Rainer Mausfeld, and Sverker Runeson for stimulating discussions and Bennett Bertenthal for providing us with the walker-generating program. We especially thank John $\mathrm{C}$. Baird for valuable advice on an earlier version of the manuscript and Arseny Sokolov for moral support. We also thank two anonymous reviewers for helpful comments. Correspondence concerning this article should be addressed to M. Pavlova, Institute of Medical Psychology and Behavioral Neurobiology/ MEG-Center, University of Tübingen, Gartenstr. 29, D-72074 Tübingen, Germany (e-mail: marina.pavlova@uni-tuebingen.de). mans and animals depicted in animated cartoons (Mitkin \& Pavlova, 1990), despite the highly reduced and unusual structural information available. By 3-5 months of age, infants can discriminate a computer-simulated pointlight walker from a similar display in which local rigidity between dots is perturbed (Bertenthal, Proffitt, \& Kramer, 1987) or from displays of identical absolute motions with scrambled spatial relations between the moving points (Bertenthal, Proffitt, \& Cutting, 1984). Even cats (Blake, 1993) and bottlenose dolphins (Herman, MorelSamuels, \& Pack, 1990) are able to perceive point-light displays.

Humans recognize biological motion despite the distortions caused by reverse transformation (showing the film backwards) or by changing the presentation rate to about 0.5 times faster or slower than normal (Pavlova, 1992, 1995). Ahlström, Blake, and Ahlström (1997) reported that observers can discriminate between canonical and phase-scrambled point-light figures, and that they tolerate variations in dot contrast and spatial frequency.

Much research has established that with upright orientation of a target, observers can detect a point-light figure embedded in a simultaneous moving-dot mask (Bertenthal \& Pinto, 1994; Cutting, Moore, \& Morrison, 1988; Mather, Radford, \& West, 1992; Neri, Morrone, \& Burr, 1998; Thornton, Pinto, \& Shiffrar, 1998). Yet, when a display was presented for less than $0.8 \mathrm{sec}$, the ability to determine the apparent direction (facing) of a camouflaged walker fell to chance level (Cutting et al., 1988).

On the other hand, it has recently been shown in a number of psychophysical and perceptual studies that display inversion dramatically impedes the perception of biological motion. Upside-down presentation prevents infants from discriminating a point-light walker from similar displays (Bertenthal et al., 1984; Bertenthal et al., 1987; Bertenthal, Proffitt, Spetner, \& Thomas, 1985). Adults 
and 3- to 6-year-olds failed in the recognition of inverted animated cartoons (Mitkin \& Pavlova, 1990; Pavlova, 1989). When presented through multiple apertures at orientations of $0^{\circ}, 90^{\circ}$, and $180^{\circ}$, only an upright-oriented walker was reliably identified (Shiffrar, Lichtey, \& Heptulla Chatterjee, 1997). Under observation through a Dove prism, even performance in such a relatively easy task as discrimination between texture-defined canonical and phase-scrambled walkers was degraded (Ahlström et al., 1997). Sumi (1984) reported that inversion of the original Johansson films led to an impression of unnatural movements even when observers were aware of the manipulation of a display or when responses were restricted to categories of human/nonhuman motion. Previous experience with upside-down displays affected their recognition very little. Furthermore, inversion of pointlight displays disrupted the ability to judge basic emotions represented in dance (Dittrich, Troscianko, Lea, \& Morgan, 1996), types of human actions (Dittrich, 1993), and the gender of a walker (Barclay, Cutting, \& Kozlowski, 1978).

Taken together, these findings raise the issue of the nature of orientation specificity in the perception of biological motion. The pattern of intrastimulus kinematics remains the same regardless of display orientation. Thus, contrary to the assumptions of the computational structurefrom-motion models (Hoffman \& Flinchbaugh, 1982; Sugie \& Kato, 1987; Webb \& Aggarwal, 1982), extracting structure from biological motion does not depend only on the relative motions of its elements defined by local rigidity or by an elongated axis of symmetry. Obviously, the perceptual system implements additional constraints for the unambiguous interpretation of biological motion. Two types of such constraints can be distinguished: (1) ecological constraints that represent sensitivity of the visual system to regularities in the environment (e.g., occlusion or gravity) and (2) knowledge-based or internal constraints, which can be defined as a prior knowledge about the outer world. Ecological constraints appear to affect primarily bottom-up processing of a point-light display, whereas knowledge-based constraints reflect a top-down influence on biological motion. Although much research has implicitly assumed that the processing constraints in the perception of complex events are closely interconnected and hierarchically nested, only a few data are available on their interrelation. For example, a priori information about display orientation was found to be insufficient for reliable recognition of an inverted walker (Pavlova, 1993).

We suppose that ecological constraints play a prominent role in event perception. Actually, displays with filmed biological motion are much less ambiguous than computer simulations. Computer-generated point-light displays usually lose the higher harmonic motions of the minor joints of the foot or natural gait asymmetry (Proffitt, Bertenthal, \& Roberts, 1984). Among other parameters, occlusion between moving dots during a walking cycle significantly improves recognition of a synthesized point-light walker and reduces its perceptual instability
(Proffitt et al., 1984). Even infants can discriminate between a computer-simulated canonical walker and a figure without occlusion (Bertenthal et al., 1985). On the other hand, the lack of occlusion in filmed biological motion is ineffective for its perception (Runeson, 1994), apparently because of redundant stimulus information in natural displays.

Dynamic constraints that reflect sensitivity to a match between event kinematics (configuration of trajectories and velocities) and dynamics (mass- and force-related information) may also limit nonveridical interpretations of biological motion. Sensitivity to dynamic properties revealed from kinematics was demonstrated when estimating the relative weight of moving balls in collisions (Flynn, 1994) or the weight of an object lifted by a pointlight person (Runeson \& Frykholm, 1983). Earlier work indirectly favors the role of dynamic constraints in event perception. Observers were able to distinguish humans in action from animated point-light puppets (Johansson, 1976). Gender discrimination in filmed displays significantly decreased with a change in presentation rate (three times slower than normal), mainly because of distortions in the perception of the gravitational force (Barclay et al., 1978; see also Mather et al., 1992). Bingham, Schmidt, and Rosenblum (1995) reported that among three viewing conditions (upright display and observer, upright display and inverted observer, and inverted display and upright observer), the last one produced the most errors in the identification of simple point-light events (such as falling leaves or a pendulum). They suggested that event recognition depends on absolute display orientation in the gravitational field, rather than on the relative orientation of the display and the observer. Much less is known, however, about how the perception of biological motion depends on such constraints. More specifically, when the natural orientation of an event is changed, does the discrepancy between perceived kinematics and dynamics influence the perception of biological motion?

The present set of four experiments, in which we used an image-plane rotation of the display, was designed to systematically study how display orientation affects the perception of biological motion. Experiment 1 was aimed at examining how spontaneous recognition of a pointlight walker varies with a gradual change in orientation. In Experiments 2 and 3, a masking paradigm was used to investigate how display orientation affects recovery of a known point-light figure. The essential difference of the task from spontaneous recognition is that it deals primarily with top-down processing of biological motion. Experiment 4 was conducted to establish whether and, if so, how a priming effect in the perception of biological motion depends on prime orientation.

\section{EXPERIMENT 1}

\section{Spontaneous Recognition of a Rotated Walker}

The primary goals of this experiment were to examine (1) how spontaneous recognition varies with a change in 
orientation and (2) whether a range of deviations from upright exists within which a display is spontaneously recognizable.

\section{Method}

Subjects. Twenty paid subjects (18-25 years of age, with an equal number of males and females) with normal or corrected-tonormal vision participated. None had previous experience with point-light displays. They were run individually.

Stimuli and Apparatus. A computer-generated biological motion display consisted of an array of 11 dots on the head and main joints of an invisible walker. The simulation program was created using an algorithm described by Cutting (1978). A walking figure was seen moving as if on a treadmill, facing right. A gait cycle was accomplished in 40 frames, with a frame duration of $36 \mathrm{msec}$. This resulted in a walking speed of about 42 complete cycles per minute, which corresponded to a normal walking speed that ranged from 30 to 70 cycles per minute (Inman, Ralston, \& Todd, 1981). With the upright orientation, a walker subtended a visual angle of $4.0^{\circ}$ in height and $2.8^{\circ}$ in width at the most extended point of a gait cycle. To avoid a framing effect, the point-light figure was displayed on a circular-screen monitor $18 \mathrm{~cm}$ in diameter. The monitor was mechanically rotated in the image plane in order to stabilize the spatial resolution of the stimuli across changes in orientation. An observer sat in a dimly illuminated room at a distance of $90 \mathrm{~cm}$ from the screen. His or her head was fixed in a head-and-chin rest.

Procedure. Two independent groups of subjects were presented with the point-light display, which was rotated in $30^{\circ}$ steps in the image plane. In both groups, orientation was varied from $180^{\circ}$ to $0^{\circ}$-for one of them, clockwise, and for the other, counterclockwise. Rotation from upright to a $180^{\circ}$ orientation was not used, because a pilot study showed a carry-nver effect: Once the display had been recognized as a walker at a particular orientation, this interpretation became dominant for the subsequent orientations. For each orientation, the viewing period was $60 \mathrm{sec}$. The observers were asked to press a key whenever one interpretation changed to another (e.g., when a bouncing or a swinging of the moving dots was seen in turn). The notion of instability was clarified by demonstrating two wellknown examples of ambiguous static pictures: Rubin's face-vase picture and the Necker cube, which are usually bistable. The observers were told that the displays might have one, two, or even more than two interpretations. Number of keypresses was used as a measure for perceptual instability. Proffitt et al. (1984) used a similar task in their study of how the occlusion of dots in a point-light display affects its perception. Decisive reasons for using such a procedure in the present study were twofold. First, it avoids making the task one of explicit identification. Second, because of the potential ambiguity of a point-light display, perceptual instability by itself appears to be an informative characteristic. After each presentation, the subjects were asked to briefly describe what they had seen and to indicate how many different interpretations of the pattern they had.

\section{Results}

Figure 1 shows that the mean number of keypresses increased as orientation varied from inverted to $90^{\circ}$ and then decreased almost entirely to upright orientation. The difference between the two groups of subjects was not significant $[F(1,9)=0.467, p>.05]$. In both groups, perceptual instability as a function of display orientation can be approximated by a parabolic curve: By using the method of orthogonal polynomials, significant linear $(p<.05)$ and quadratic $(p<.05)$ trends were found. As can be seen in Figure 1, the peak of instability occurred at $90^{\circ}$ orientation. An analysis of variance (ANOVA) indi- cated a highly significant effect of display orientation on perceptual instability $[F(6,9)=7.154, p<.01]$. Display orientation also affects the number of display interpretations $[F(6,9)=7.759, p<.01]$. However, these measures of performance varied independently with a change in orientation $[F(6,9)=4.308, p<.05$; Figure $1 \mathrm{~B}]$. This indicates that the effect of orientation on instability was caused by perceptual switching from one interpretation to the other, rather than simply by holding a number of different interpretations.

Inspection of Figure 2 indicates that spontaneous walker recognition improved abruptly with changing orientation. Each data point of this figure corresponds to the percentage of subjects who, at least once, interpreted a display at a particular orientation as a walking figure. Despite the relatively long viewing period, at orientations of $150^{\circ}, 120^{\circ}$, and $90^{\circ}$, the pattern was very rarely perceived as a walker. Instead, the display was described in a variety of ways, ranging from "swinging of dots back and forth" to "rotation of a stick or a hand." In a couple of cases, when presented with a $90^{\circ}$ orientation, it was interpreted as a very strange swimmer or drummer.

When the display was presented at $60^{\circ}$ and $30^{\circ}$ orientations, most observers reported seeing the walking figure. However, with the $60^{\circ}$ orientation, the mean response time (RT) to a first impression of a walker was about $19 \mathrm{sec}$, which is much longer than that usually reported for the perception of upright filmed or synthesized pointlight displays (Johansson, 1976; Mitkin \& Pavlova, 1990; Perrett, Harries, Benson, Chitty, \& Mistlin, 1990). As was expected from the pilot study, the results showed a carryover effect regarding walker recognition: Once the display was recognized as a walker at a particular orientation, this interpretation became dominant or was preserved for the ensuing orientations.

\section{Discussion}

The results of Experiment 1 show that with changing orientation from inverted to upright, spontaneous recognition of a point-light walker improves abruptly. Orientations from inverted to $90^{\circ}$ dramatically impeded spontaneous recognition despite the much longer viewing period than that usually needed for veridical perception of upright displays (Johansson, 1976; Pavlova, 1992; Shiffrar et al., 1997).

However, starting from $60^{\circ}$ and $30^{\circ}$ orientations, most observers reported seeing a point-light walker. These findings suggest that the power of dynamic constraints is graded, or in other words, their effectiveness in event recognition is limited. When a display is rotated in the image plane, in contrast with filmed down- or uphill movement of a real walker, there is some mismatch between perceived kinematics and dynamics. However, within a limited range of deviations from upright orientation, such a mismatch does not appear to substantially impair bottom-up processing of biological motion.

These results are consistent with the findings reported by Pavlova (1992). Reverse transformation (showing the 


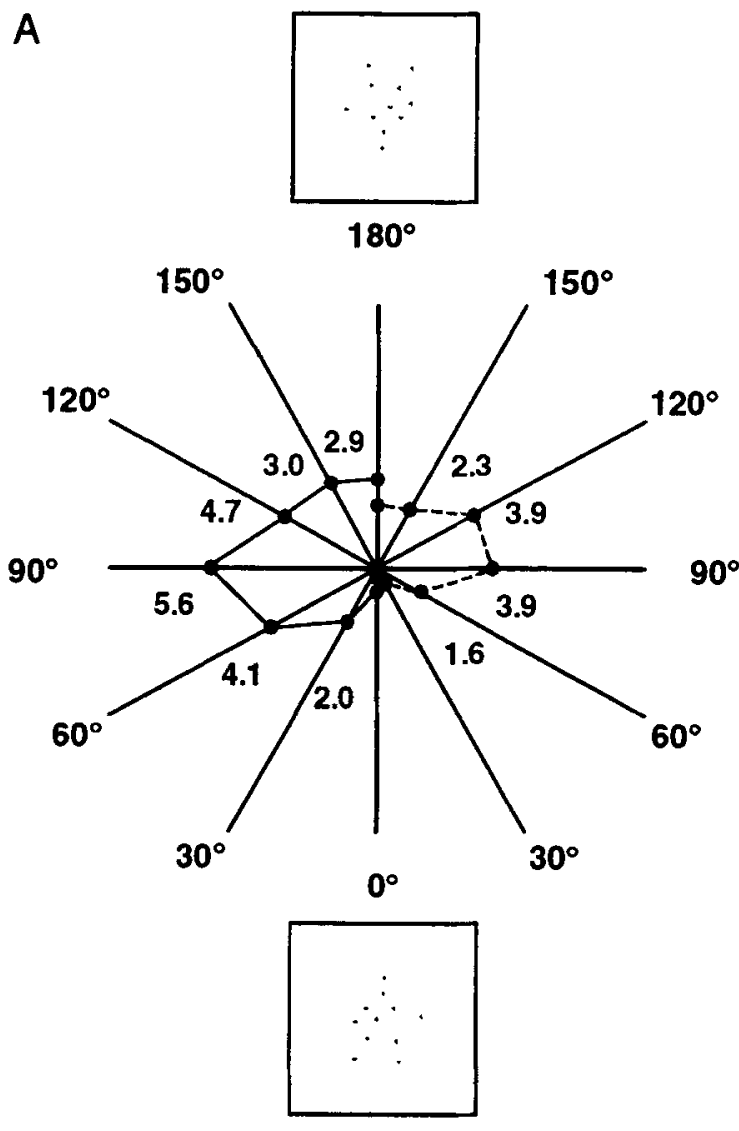

B

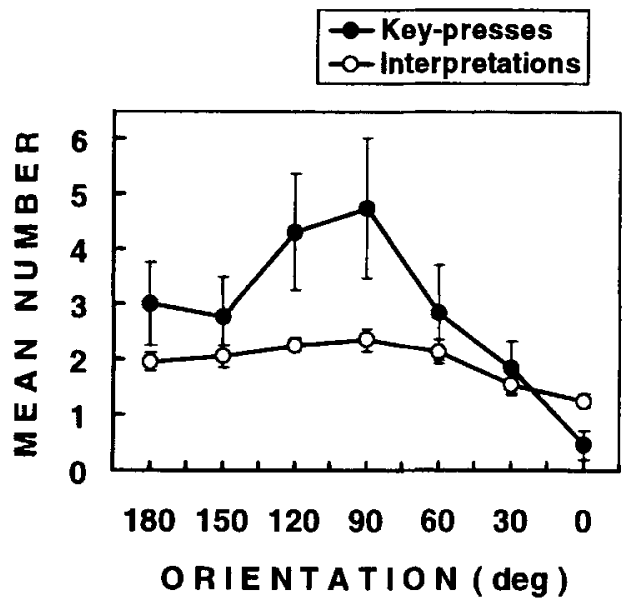

Figure 1. The results of Experiment 1. (A) Perceptual instability (the mean number of keypresses during the viewing period) as a function of display orientation. The solid line represents the data for one group of subjects, the dashed line for the other. Each experimental point is based on data from 10 subjects. (B) Perceptual instability (closed circles) versus the number of different interpretations (open circles). Vertical bars show $\pm S E$. Each experimental point is based on data from 20 subjects.

film backward) was expected to cause a discrepancy between event kinematics and natural dynamics, which affects perception of biological motion. Instead, adults and 6-year-olds readily extracted an invariant structure in up- right-oriented displays presented either as usual or in reverse transformation. Recognition of a display was mainly determined by the apparent direction of common motion of a point-light quadruped. For example, the display that was recognized as a cat moving to the right with tail lifted was interpreted as a giraffe moving to the left when the film was shown backward. These findings suggest that in interpreting biological motion, the perceptual system may neglect a mismatch of kinematics and dynamics, presumably in favor of more ecologically or functionally meaningful factors. Other measures of performance, however, indicated that observers are sensitive to this mismatch. For example, RT to a point-light display presented backward was systematically longer (Pavlova, 1992). Likewise, in the present experiment, with orientations of $30^{\circ}$ and $60^{\circ}$, the display was perceived as unstable. Given the strong carry-over effect, which was observed across a change in orientation, the perceptual instability with these orientations appears to be fairly remarkable. One might argue that this effect was simply induced by a self-instruction to perceive a display as unstable or by experience with preceding orientations with which instability was high. Yet, our data contradict these arguments. When the display was finally presented upright, the perceptual instability was close to zero (see Proffitt et al., 1984).

\section{EXPERIMENT 2 Detection of a Point-Light Walker Within a Mask}

In Experiment 2, a masking paradigm was used to examine how orientation affects recovery of a camouflaged point-light walker. Because a simultaneous mask is supposed to impair bottom-up processing of biological motion (see, e.g., Bertenthal \& Pinto, 1994; Thornton et al., 1998 ), in order for a camouflaged figure to be detected, observers must be familiar with it. The essential difference of the task from spontaneous recognition is that it deals primarily with top-down processing of biological motion, although bottom-up and top-down processes usually are closely interconnected and probably cannot be completely isolated.

Cutting et al. (1988) reported that one of the most effective masks to camouflage a walker's facing is composed of spatially scrambled dots on the joints of a walker. Their pilot study showed that even such masks as these were almost ineffective in camouflaging an upright figure. Bertenthal and Pinto (1994) found, however, that observers failed to detect a camouflaged walking figure and to judge its facing when the display was presented upside down. In this experiment, we examined sensitivity to a camouflaged walker, which was rotated in the image plane over a range between upright and inverted displays.

\section{Method}

Subjects. Seven subjects ( 2 women and 5 men; mean age, 31.2 years), including both authors, with normal or corrected-tonormal vision were recruited as volunteers to participate in the experiment. They were run individually. 


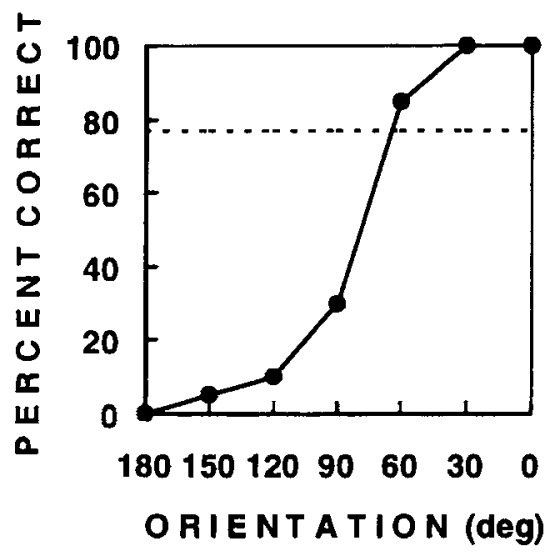

Figure 2. Percentage of walker recognition as a function of display orientation (Experiment 1). The dashed line represents the threshold probability for significance at the $5 \%$ level according to a $Z$ criterion.

Apparatus and Stimuli. Two types of stimuli were used (see Figure 3). One of them (the target) represented an 11-dot point-light walker, the characteristics (size, speed, etc.) of which were the same as those in Experiment 1. This figure was simultaneously camouflaged by a mask of 66 moving dots that corresponded to spatially scrambled points on the joints of a walker. Thus, the mask shared the same particular parameters of motion as those of the canonical point-light figure. The masking level, defined as the number of moving dots, was chosen so as to enable a comparison of the data with the findings of Bertenthal and Pinto (1994). The other type of stimuli (noise) was a 77-dot mask alone. For the generation of stimuli, a computer simulation program was created, using the algorithms described by Cutting (1978; Cutting et al., 1988). In both stimulus displays, moving dots were distributed within a region about $5.0^{\circ}$ in height by $6.8^{\circ}$ in width. The viewing period was about $1 \mathrm{sec}$, which was comparable with the overall display duration used by Bertenthal and Pinto. The experimental setup was essentially the same as that in Experiment 1.

Procedure. At five display orientations between upright and inverted $\left(0^{\circ}, 45^{\circ}, 90^{\circ}, 150^{\circ}\right.$, and $\left.180^{\circ}\right)$, the observers saw a sequence of displays. Half of the displays consisted of a camouflaged pointlight walker, whereas the other half were only masks. The observers participated in seven experimental sessions (for about $40 \mathrm{~min}$ each) with five randomly presented blocks - 30 trials for each orientation. With each orientation, the observers completed a total of 210 trials. Each block was preceded by a 3-sec exposure to the same oriented point-light figure without a mask. In a confidence-rating procedure, the observers had to judge whether a walker was present. A 5-point equal-spaced unipolar scale was used (5, from $100 \%$ to $80 \%$ confident in the presence of a walker; 4 , from $80 \%$ to $60 \% ; 3$, from $60 \%$ to $40 \% ; 2$, from $40 \%$ to $20 \%$; and 1 , from $20 \%$ to $0 \%$ ). No feedback was given regarding the subject's performance.

\section{Results}

To compare detectability for a point-light walker as a function of display orientation, we pooled individual data from the 7 observers by averaging the frequencies with which each observer gave each rating. Figure 4 shows the receiver-operating characteristic (ROC) curves obtained for each orientation. A jackknife procedure was employed to calculate statistically unbiased parameters of ROC curves from pooled rating-method data (Dorfman \& Ber- baum, 1986). For further data processing, the jackknife estimation of the area under the ROC curve, $A_{z}$, was taken as a sensitivity index (Figure 5).

A one-way ANOVA performed on individual values of $A_{z}$ revealed a highly reliable effect of display orientation on detectability $[F(4,6)=16.476, p<.01]$. Sensitivity was already reduced at $45^{\circ}$ orientation. The difference between $A_{z}$ s for the upright and the $45^{\circ}$ orientations was highly significant $[t(6)=4.549, p<.01]$. Figure 4 shows that the ROC curves for orientations of $90^{\circ}, 135^{\circ}$, and $180^{\circ}$ are situated close to one another. We did not find any significant difference in sensitivity between these orientations.

Despite the decrease with image-plane rotation, sensitivity with all the orientations exceeded chance level. This was assessed using the $\chi^{2}$-statistic, which involved both the sensitivity index and the slope of the binormal ROC curve (Metz \& Kronman, 1980). The lowest $\chi^{2}$ value was for the $135^{\circ}$ orientation and was equal to 23.447 $(p<.01)$.

\section{Discussion}

The main finding of this experiment was that despite prior familiarization with a point-light figure, at each particular orientation its detection declined with image-plane rotation. Moreover, this decrease was nonmonotonic: With $90^{\circ}-180^{\circ}$ orientations, sensitivity to a camouflaged figure was essentially the same.

High sensitivity to a camouflaged point-light walker with upright orientation corresponds to earlier findings (Bertenthal \& Pinto, 1994; Cutting et al., 1988; Mather et al., 1992; Neri et al., 1998; Thornton et al., 1998). A significant difference in detectability between upright and inverted displays also agrees with Bertenthal and Pinto's results. Unlike Bertenthal and Pinto (Experiment 1), however, we found that even with inversion, observers were sensitive to the presence of a camouflaged figure. The discrepancy between our results and those obtained by Bertenthal and Pinto can be attributed to some methodological differences (e.g., two vs. five display orientations or $2 \mathrm{AFC}$ vs. confidence-rating procedures). One difference of possible importance is the number of trials: 100 versus 210 in our study. To control for this possibility, we performed the data analysis with only the first 90 trials. With all the orientations, sensitivity was significantly lower, but it exceeded chance. In the work by Bertenthal and Pinto, the procedure of familiarization with an unmasked walker was not explicitly described. Keeping in mind that in order for a camouflaged figure to be detected, subjects must know which target they have to look for, we suggest that the familiarization phase might substantially influence detectability.

In performing the task, a subject was free to use whatever strategy he/she preferred. Despite or probably even owing to the mask complexity, the observers could extract the most salient event parameter to facilitate performance. Presumably, in some cases, they did not need to recover the whole fine structure. One possibility is that 

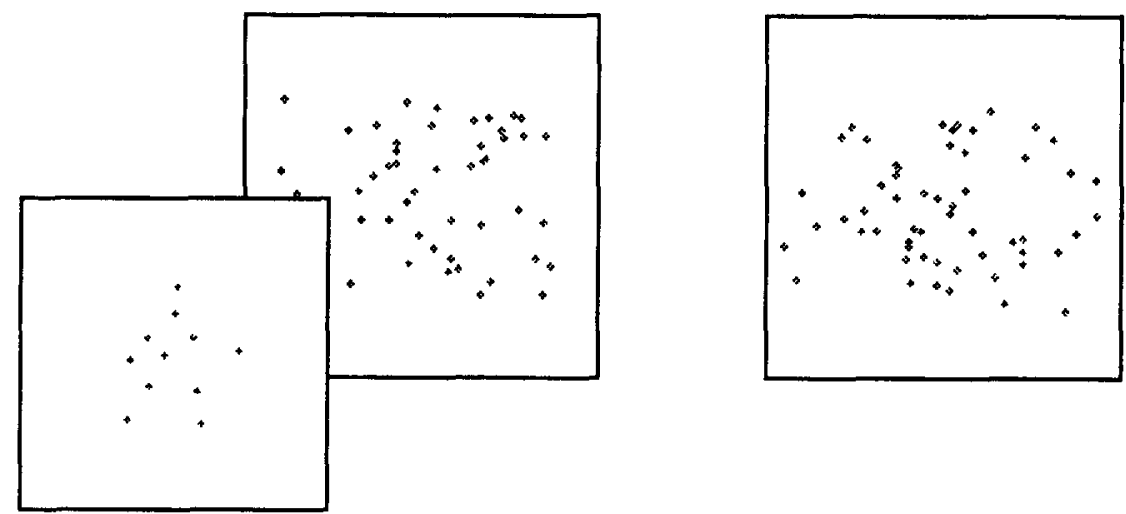

Figure 3. The left panel depicts a static frame from a walking cycle of a canonical pointlight figure. The points represent the locations of the major joints. A 66-dot moving mask simultaneously camouflaged the 11-dot walker. The right panel depicts a 77-dot mask alone. The displays are represented in reverse contrast: Observers were shown patterns consisting of bright moving dots against a dark background.

the observers detected a walking figure only as a global cloud of moving dots. To eliminate this possibility, in Experiment 3 , we included a partly distorted walker embedded in a noise display. We supposed that observers have to perceive hierarchical connections between moving dots in a canonical figure in order to discriminate between displays with canonical and distorted walkers.

\section{EXPERIMENT 3 Canonical Versus Distorted Walker}

\section{Method}

Subjects. Seven subjects ( 1 woman and 6 men, 23-44 years of age) with normal or corrected-to-normal vision were recruited as volunteers. They were run individually.

Stimuli and Procedure. The experimental setup, stimuli, and procedure were essentially the same as those in Experiment 2 . The only difference was that a partly distorted walking figure was embedded in a noise display. Pairwise relations between moving dots on the arms of this figure were perturbed (Figure 6) by setting the parameter $D$ (shoulder excursion as a multiple of hip) of a walkergenerating program to 15 instead of 1.5 for the canonical figure. A 66-dot mask camouflaged both the canonical and the distorted figures. As in the previous experiment, prior to each experimental block, the observers were exposed (for $3 \mathrm{sec}$ ) to a similarly oriented noncamouflaged canonical walker, but not to a distorted one. On each trial, the subjects had to judge whether a canonical walker was present.

\section{Results}

Figure 4 shows the ROC curves obtained for each display orientation. The jackknife estimations of the area under the ROC curve (Figure 5) indicate that sensitivity decreased nonmonotonically with varying orientation from $0^{\circ}$ to $135^{\circ}$ and then increased slightly at $180^{\circ}$. A oneway ANOVA revealed a significant effect of display orientation on detectability $[F(4,6)=8.04, p<.01]$.

As in Experiment 2, detectability was already reduced at the $45^{\circ}$ orientation. The difference between $A_{z} \mathrm{~s}$ for upright and $45^{\circ}$ orientations was significant $[t(6)=2.721$, $p<.05$ ]. In turn, at the $45^{\circ}$ rotation, sensitivity was significantly higher than that at the $90^{\circ}, 135^{\circ}$, and $180^{\circ}$ ori- entations $[t(6)=5.112,3.812$, and 4.034 , respectively, $p<.05]$. We did not find any difference in sensitivity between $90^{\circ}, 135^{\circ}$, and $180^{\circ}$.

A two-way ANOVA indicated that with all the display orientations, sensitivity was systematically lower than in Experiment $2[F(4,6)=6.41, p<.05]$. The interaction of experiment $\times$ orientation was not significant $[F(4,6)=$ $0.707, p>.05]$ : The curves representing sensitivity to a camouflaged walker in Experiments 2 and 3 are parallel to one another (Figure 5).

Yet, with all the orientations, performance exceeded chance level. The lowest value of $\chi^{2}$ was for the $135^{\circ}$ orientation $\left[\chi_{2}^{2}=26.55, p<.05\right]$. The effect of orientation on detectability was most pronounced within the first 90 trials, although sensitivity was always above chance. The lowest value of $\chi^{2}$ was for $135^{\circ}\left[\chi_{2}^{2}=7.588, p<.05\right]$. Learning to detect a walker proceeded more rapidly for $0^{\circ}$, $45^{\circ}$, and $90^{\circ}$ than for $135^{\circ}$ and $180^{\circ}$ : Differences in sensitivity between the first 90 and the final 120 trials were significant for these orientations $[t(6)=4.561,3.474$, and 2.834, respectively, $p<.05]$.

\section{Discussion}

Experiment 3 produced basically the same pattern of results as Experiment 2. Despite prior familiarization with point-light figures, detectability of a camouflaged walker nonmonotonically leveled off with changing orientation. However, it remained above chance even at $135^{\circ}$. Given the close similarity of the target and the noise displays, the high sensitivity to the canonical figure appears amazing. Yet, it should be stressed that in both Experiments 2 and 3 , the observers had to detect known patterns. In this case, recovery of the coherent structure is connected primarily with top-down processing of biological motion. A comparison with the findings of Experiment 1 , which showed that with orientations between $180^{\circ}$ and $90^{\circ}$, the observers failed in spontaneous recognition of a point-light walker, suggests that orientation influences bottom-up processing of biological motion 


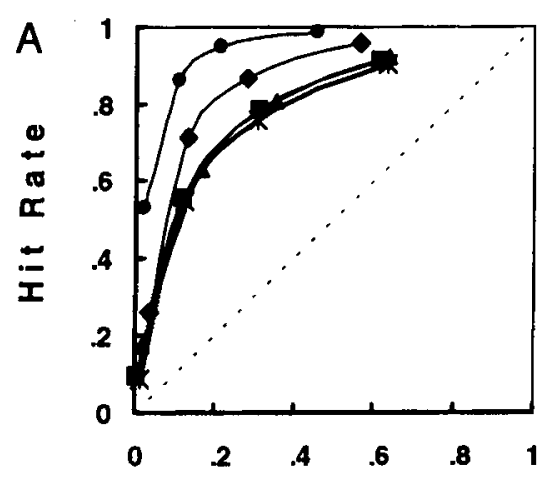

False-Alarm Rate

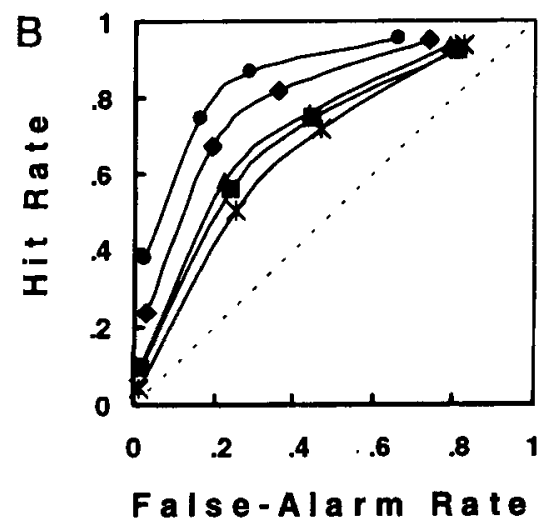

Figure 4. Receiver-operating characteristic curves obtained in (A) Experiment 2 and (B) Experiment 3. Data are represented by circles for the upright display orientation, diamonds for $45^{\circ}$, triangles for $90^{\circ}$, asterisks for $135^{\circ}$, and squares for $180^{\circ}$.

more strongly than it influences top-down processing. At the same time, detection of a known structure is affected by orientation in similar ways, as are some characteristics of spontaneous perception (e.g., perceptual stability). Both detectability and perceptual stability decreased with a change of orientation from upright to $90^{\circ} / 135^{\circ}$ and then slightly increased up to display inversion (see Figures 1 and 5).

The results suggest that processing constraints in the perception of biological motion, which are connected with orientation (e.g., an elongated axis of symmetry or dynamic constraints), are hierarchically nested (see also Pavlova, Sokolov, \& Bülthoff, 1998). Although with image-plane rotation, dynamic constraints lose their strength, other processing constraints become more powerful. For instance, the lower sensitivity at a $135^{\circ}$ than at a $180^{\circ}$ rotation may be accounted for by an axis-ofsymmetry constraint that is implemented by the visual system at $180^{\circ}$. Likewise, it seems that owing to the inefficiency of this constraint, perceptual instability of a point-light walker was higher at $90^{\circ}-150^{\circ}$ than at $180^{\circ}$ (Experiment 1). It is noteworthy that in both Experiments 2 and 3 , sensitivity was lower at the $135^{\circ}$ orientation than at inversion, despite the lack of everyday experience with both of them. However, this observation requires special empirical clarification.

The first three experiments of this series were concerned primarily with either bottom-up or top-down processing of biological motion. Experiment 4 was designed to examine in more detail the interrelation between topdown influence on biological motion and the constraints connected with display orientation.

\section{EXPERIMENT 4 \\ Priming in Detection of a Camouflaged Walker}

In Experiment 4, in addition to image-plane rotation of a camouflaged walker, we implemented a long-term priming paradigm. This paradigm implies that prior viewing of a stimulus facilitates performance later on. We hypothesized that if ecological constraints play a prominent role in biological motion processing, a priming effect would occur only with an upright-oriented prime. Alternatively, if knowledge-based constraints were decisive, a priming effect should be observed with all the prime orientations. Thus, Experiment 4 was conducted to establish whether a priming effect in the perception of biological motion depends on prime orientation.

\section{Method}

Subjects. The subjects were 25 paid volunteers (18-32 years of age) with normal or corrected-to-normal vision. They were recruited from the Max Planck Institute subjects pool. None had previous experience with these types of displays. The observers were tested individually.

Stimuli and Procedure. The experimental setup, stimuli, and procedure were essentially the same as those in Experiment 2. On a trial, a subject had to press as rapidly as possible a "yes/no" key to indicate whether the walker was present. Following this, they gave a confidence rating on a 6-point equal-spaced unipolar scale (6, absolutely confident; 1 , absolutely unconfident). The experiment consisted of three sessions of five randomly presented blocks-32 trials for each orientation. The key difference between this experiment and Experiment 2 was that prior to a block of trials, the subjects were primed (for $10 \mathrm{sec}$ ) with a sample of a noncamouflaged walker that was upright, oriented $45^{\circ}$ or $90^{\circ}$, or inverted.

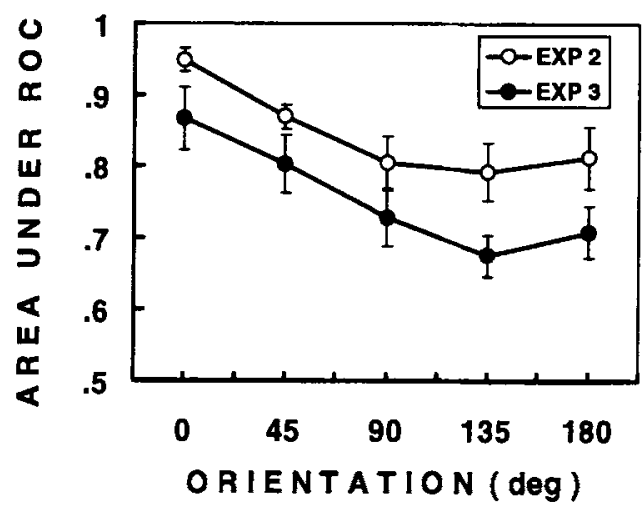

Figure 5. Jackknife estimates of the area under the ROC curves obtained in Experiments 2 (open circles) and 3 (closed circles). Vertical bars show $\pm S E$. 

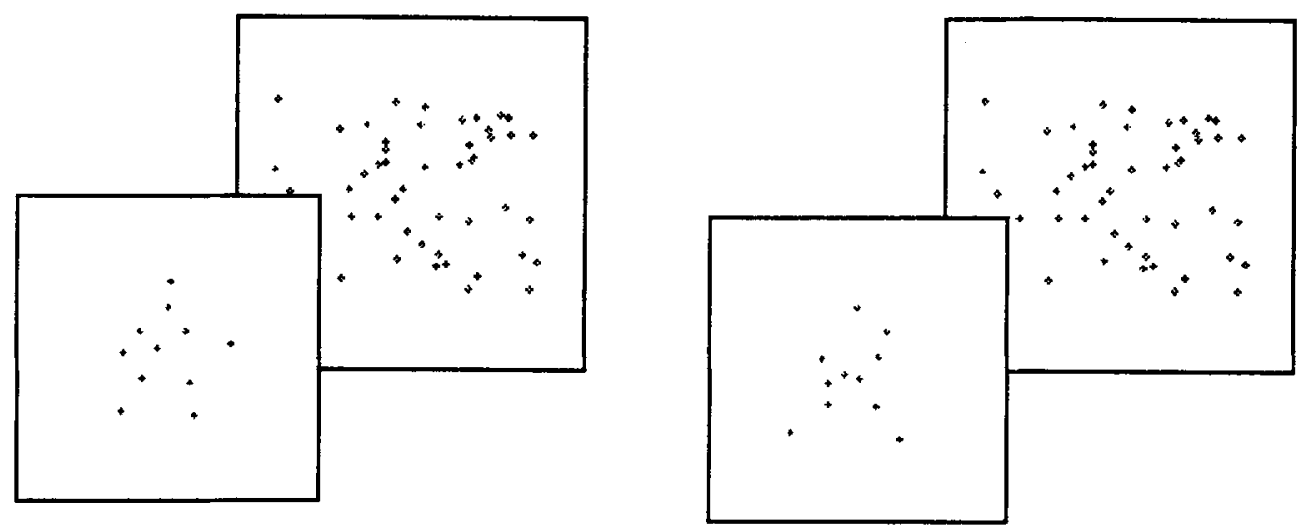

Figure 6. Representation of the stimuli used in Experiment 3. The left panel depicts a static frame from a walking cycle of a canonical point-light figure; the right panel depicts a partly distorted walking figure. Both figures were simultaneously camouflaged with a 66-dot moving mask.

Thus, each of four groups of subjects saw a point-light walker without a mask in one of these particular orientations. The observers were explicitly informed about the relative orientations of the priming and the primed displays. Except for the group that was primed with a $90^{\circ}$-oriented walker ( 7 subjects), all the groups consisted of 6 observers.

\section{Results}

Figure 7 shows the ROC curves obtained with different-oriented primes. As can be seen there, a pronounced priming effect occurred only with the upright-oriented prime: The ROC curve for the same-oriented display is situated higher than those for other orientations. The jackknife estimates of the area under the ROC curve, $A_{z}$, are shown in Figure 8.

In the group primed with an upright-oriented walker, the highest sensitivity was found for similarly oriented displays. A one-way ANOVA revealed a reliable effect of display orientation on sensitivity: It decreased with increasing deviation of the display from the upright orientation $[F(4,5)=7.827, p<.05]$. A within-group analysis showed highly significant differences between $A_{z} \mathrm{~s}$ for primed and nonprime orientations $[t(5)=8.889,9.676$, 9.676 , and $17.881, p<.05$, between $0^{\circ}$ and $45^{\circ}, 90^{\circ}, 135^{\circ}$, and $180^{\circ}$, respectively].

In the group primed with the $45^{\circ}$-oriented walker, we also found a significant effect of display orientation on detectability $[F(4,5)=7.412, p<.05]$. Sensitivity was statistically the same for $45^{\circ}$ - and upright-oriented displays but higher than for all the other orientations. In the groups primed with $90^{\circ}$ - and $180^{\circ}$-oriented walkers, an ANOVA did not indicate any significant difference in sensitivity $[F(4,5)=0.132$ and $0.264, p>.05$, respectively $]$. Figure 7 shows that the ROC curves for all orientations are situated very close to each other.

A between-group analysis showed that sensitivity to upright displays was higher with the same-oriented prime than with the other primes (Figure 8). A one-way ANOVA revealed a decrease of sensitivity to upright-oriented dis- plays with increasing deviation of the prime from the upright orientation $[F(3,5)=5.444, p<.05]$. We did not find any significant difference in sensitivity to $45^{\circ}$-, to $90^{\circ}$-, and to $180^{\circ}$-oriented displays as a function of prime orientation $[F(3,5)=2.046,0.077$, and 1.045 , respectively, $p>.05]$. For $135^{\circ}$-oriented displays, sensitivity was also independent of primes $[F(3,5)=1.52, p>.05]$, although we did not use a specifically oriented one.

We also performed an analysis of RTs for correct responses (hits only). For each subject and experimental condition, we processed only those RT values that did not exceed the cutoff value of three standard deviations from the mean RT (Figure 9). A two-way ANOVA did not reveal any differences in $\mathrm{RT}$ as a function of prime $[F(3,5)=$ $0.542, p>.05]$ or display orientation $[F(4,5)=2.572$, $p>.05]$.

\section{Discussion}

The findings of Experiment 4 clearly indicate that only an upright-oriented prime strongly affects detection of a camouflaged point-light walker: It significantly improves performance for the same-oriented displays. It is fairly remarkable that $90^{\circ}$ - and $180^{\circ}$-oriented primes did not facilitate performance for the similarly oriented displays. This evidence provides strong support in favor of the primacy of ecological constraints in biological motion processing.

At first glance, contrary to our initial hypothesis, a long-term priming effect was observed with a $45^{\circ}$ oriented prime. Yet, this finding conforms to the data of Experiment 1, which showed that despite a mismatch between event kinematics and natural dynamics at $30^{\circ}$ and $60^{\circ}$ rotation, the display was spontaneously recognizable. However, sensitivity to upright-oriented displays in this experiment was significantly lower with a $45^{\circ}$ prime than with the upright one. This suggests that stimulus information, which is recovered at the $45^{\circ}$ orientation, does not induce as high performance as with an upright display. 


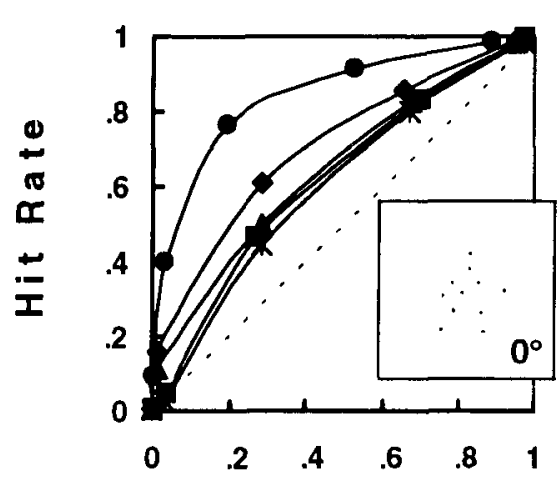

False-Alarm Rate

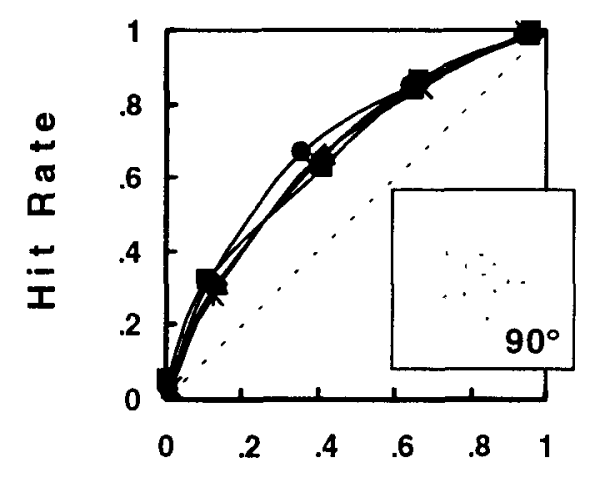

False-Alarm Rate

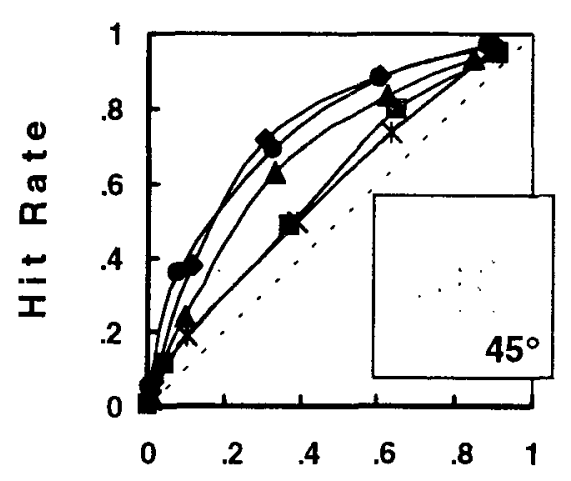

False-Alarm Rate

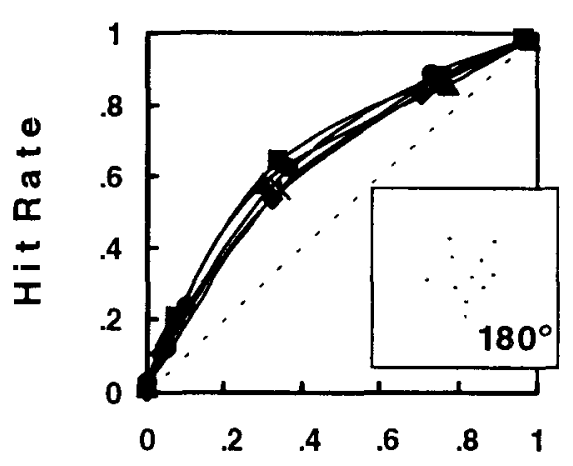

False-Alarm Rate

Figure 7. The results of Experiment 4: the receiver-operating characteristic curves obtained in four independent groups of subjects that were primed with $0^{\circ}-, 5^{\circ}-, 90^{\circ}$-, and $180^{\circ}$-oriented walkers. Data are represented by circles for the upright display orientation, diamonds for $45^{\circ}$, triangles for $90^{\circ}$, asterisks for $135^{\circ}$, and squares for $180^{\circ}$.

The present findings indicate that a long-term priming effect in biological motion occurs only if a prime corresponds to a limited range of deviations from the upright orientation within which a display is spontaneously recognizable. The only earlier work on priming in biological motion concerns short-term effects for a point-light walking figure rotated in depth (Verfaillie, 1993). For the first time, we established a differential priming effect in biological motion, which depends on image-plane display orientation. Contrary to common belief based on imageplane rotation of static objects with explicit structure (e.g., Jolicoeur, 1988), we found that the priming effect in biological motion is partly independent of the relative orientation of priming and primed displays. Moreover, an RT analysis indicated that the priming effect is not connected with a process of mental rotation or normalization. If such dependence did exist, one would expect an increase in RT with increasing relative orientation between priming and primed displays. This was not the case in our study.

\section{GENERAL DISCUSSION}

In a set of four experiments, we demonstrated that the perception of biological motion was nonmonotonically affected by a change in display orientation. Specifically, with $90^{\circ}-180^{\circ}$ orientations, (1) spontaneous recognition was dramatically impeded (Experiment 1), (2) sensitivity to a camouflaged figure was essentially the same (Experiments 2 and 3 ), and (3) the primes corresponding to these orientations did not improve detectability of a pointlight walker embedded within a mask (Experiment 4). With upright orientation, however, all the observers reported seeing a walking figure, which was perceived as stable and unambiguous. Despite prior familiarization with a rotated figure at each particular orientation, the highest sensitivity to camouflaged walker was observed with an upright display. Moreover, only an upright-oriented prime yielded a pronounced priming effect.

The data suggest that dynamic constraints in event recognition are graded in their influence. When a display is rotated in the image plane, in contrast with the filmed down- or uphill movement of a real walker, there is some mismatch between perceived kinematics and dynamics. Within a limited range of deviations from the upright orientation $\left(30^{\circ}\right.$ and $\left.60^{\circ}\right)$, such a mismatch does not appear to substantially impair recognition of biological motion. Accordingly, a long-term priming effect (Experiment 4) was observed not only with an upright-oriented prime, 


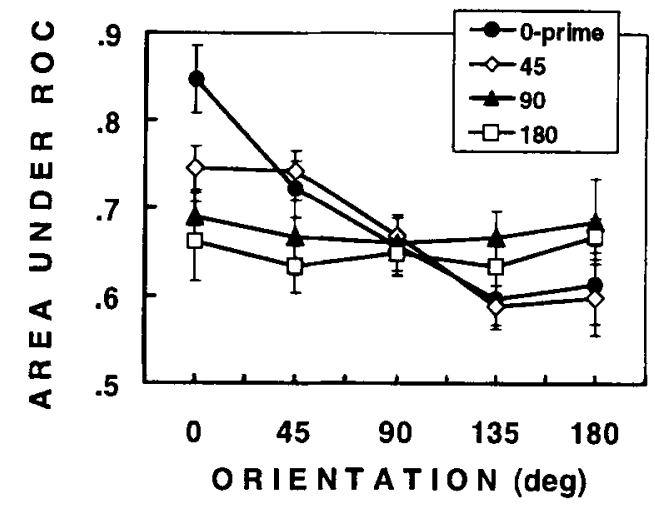

Figure 8. Jackknife estimates of the area under the receiveroperating characteristic (ROC) curves in Experiment 4. Closed circles represent values obtained in the group with $0^{\circ}-$, open diamonds with $45^{\circ}$-, closed triangles with $90^{\circ}$-, and closed squares with $180^{\circ}$-oriented primes. Vertical hars show $\pm S E$.

but also with a prime rotated $45^{\circ}$. However, sensitivity to upright-oriented displays was significantly lower with the $45^{\circ}$ prime than with the upright one. This suggests that stimulus information, which is recovered at $45^{\circ}$ rotation, does not provide as high performance as with an upright orientation. Likewise, perceptual instability of a display rotated $30^{\circ}$ and $60^{\circ}$, which was observed in Experiment 1 , indicated that observers are sensitive to the mismatch between event kinematics and dynamics that occurs with these orientations. The latter findings provide additional support in favor of constraints connected with display orientation in the perception of biological motion.

For the first time, we established a differential longterm priming effect in biological motion, which depends on image-plane display orientation. In Experiment 4, it occurred only when a prime corresponded to a limited range of deviations from the upright orientation, within which the display is spontaneously recognizable. Consid-

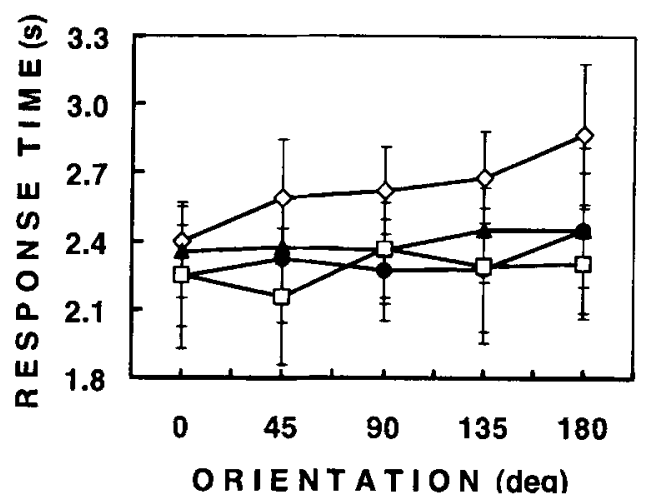

Figure 9. Response time for correct responses (hits only) as a function of prime and display orientations (Experiment 4). Closed circles represent values obtained in the group with $0^{\circ}$ oriented primes, open diamonds those with $45^{\circ}$-oriented primes, closed triangles those with $90^{\circ}$-oriented primes, and closed squares those with $180^{\circ}$-oriented primes. Vertical bars show $\pm S E$. ered as a within-subjects priming design, Experiments 2 and 3 clearly show that despite prior familiarization with a noncamouflaged walker, detectability is reduced from upright to $90^{\circ}-180^{\circ}$. Verfaillie (1993) documented shortterm priming, which occurred only when upright priming and primed walking figures shared the same in-depth orientation. The effect was not suppressed when priming and primed walkers differed in their position in the visual field and the starting position in the step cycle. His data suggest that viewpoint-specific constraints are more powerful than position cues or, in other words, that a hierarchy of orientation- and position-dependent cues occurs in biological motion. Likewise, the constraints connected with image-plane orientation were observed to be much more powerful than other constraints, such as position cues or occlusion (see, e.g., Bertenthal et al., 1985; Heptulla Chatterjee, Freyd, \& Shiffrar, 1996; Mather et al., 1992). The present data converge with observations that a priming effect does not occur for photographs depicting unnatural human poses that are impossible to perform (Daems \& Verfaillie, 1999) or for distorted dynamic actions (Nilsson, Olofsson, \& Nyberg, 1992; Olofsson, Nyberg, \& Nilsson, 1997). Kourtzi and Shiffrar (1999) also have reported that although apparent motion facilitates linkage of multiple views of a human body, priming for these views is restricted by biomechanical constraints. We did not observe priming effects with the displays representing such unnatural events as walking upside down or walking on a cliff in the same manner as with an upright orientation in the gravitational field. Taken together, our findings show that top-down influence on the perception of biological motion is limited by display orientation.

\section{REFERENCES}

Ahlström, V., Blake, R., \& Ahlström, U. (1997). Perception of biological motion. Perception, 26, 1539-1548.

Barclay, C. D., Cutting, J. E., \& Kozlowski, L. T. (1978). Temporal and spatial factors in gait perception that influence gender recognition. Perception \& Psychophysics, 23, 145-152.

Bertenthal, B. I., \& Pinto, J. (1994). Global processing of biological motions. Psychological Science, 5, 221-225.

Bertenthal, B. I., Proffitt, D. R., \& Cutting, J. E. (1984). Infants sensitivity to figural coherence in biomechanical motions. Journal of Experimental Child Psychology, 37, 213-230.

Bertenthal, B. I., Proffitt, D. R., \& Kramer, S. (1987). Perception of biomechanical motions by infants: Implementation of various processing constraints. Journal of Experimental Psychology: Human Perception \& Performance, 4, 577-585.

Bertenthal, B. I., Proffitt, D. R., Spetner, N. B., \& Thomas, A. (1985). Development of the perception of biomechanical motions. Child Development, 56, 531-543.

Bingham, G. P., Schmidt, R. C., \& Rosenblum, L. D. (1995). Dynamics and the orientations of kinematic forms in visual event recognition. Journal of Experimental Psychology: Human Perception \& Performance, 21, 1473-1493.

Blake, R. (1993). Cats perceive biological motion. Psychological Science, 4, 54-57.

Cutting, J. E. (1978). A program to generate synthetic walkers as dynamic point-light displays. Behavior Research Methods \& Instrumentation, 10, 91-94.

Cutting, J. E., MoOre, C., \& Morrison, R. (1988). Masking the motions of human gait. Perception \& Psychophysics, 44, 339-347. 
Daems, A., \& Verfaillie, K. (1999). Viewpoint-dependent priming effects in the perception of human actions and body postures. Visual Cognition, 6, 665-693.

Ditrrich, W. H. (1993). Action categories and the perception of biological motion. Perception, 22, 15-22.

Dittrich, W. H., Troscianko, T., Lea, S. E. G., \& Morgan, D. (1996). Perception of emotion from dynamic point-light displays represented in dance. Perception, 25, 727-738.

Dorfman, D. D., \& Berbaum, K. S. (1986). RSCORE-J: Pooled ratingmethod data: A computer program for analyzing pooled ROC curves. Behavior Research Methods, Instruments, \& Computers, 18, 452-462.

FLYNN, S. B. (1994). The perception of relative mass in physical collisions. Ecological Psychology, 6, 185-204.

Heptulla Chatterjee, S., Freyd, J. J., \& Shiffrar, M. (1996). Configural processing in the perception of apparent biological motion. Journal of Experimental Psychology: Human Perception \& Performance, 22, 916-929.

Herman, L. M., Morel-Samuels, P., \& Pack, A. A. (1990). Bottlenosed dolphin and human recognition of veridical and degraded video displays of an artificial gestural language. Journal of Experimental Psychology: General, 119, 215-230.

Hoffman, D. D., \& Flinchbaugh, B. E. (1982). The interpretation of biological motion. Biological Cybernetics, 42, 195-204.

INMAN, V. T., RALSTON, H., \& TODD, F. (1981). Human walking. Baltimore: Williams \& Wilkins.

Johansson, G. (1973). Visual perception of biological motion and a model for its analysis. Perception \& Psychophysics, 14, 201-211.

JoHANSSON, G. (1976). Spatio-temporal differentiation and integration in visual motion perception. Psychological Research, 38, 379-393.

JolicoeUr, P. (1988). Mental rotation and the identification of disoriented objects. Canadian Journal of Psychology, 42, 461-478.

Kourtzi, Z., \& ShIFfrar, M. (1999). Dynamic representations of human body movement. Perception, 28, 49-62.

MATHER, G., RADFORD, K., \& WEST, S. (1992). Low-level processing of biological motion. Proceedings of the Royal Society of London: Series $B, 249,149-155$.

Mather, G., \& WEST, S. (1993). Recognition of animal locomotion from dynamic point-light displays. Perception, 22, 759-766.

Metz, C. E., \& Kronman, H. B. (1980). Statistical significance tests for binormal ROC curves. Journal of Mathematical Psychology, 22, $218-243$

Mitkin, A. A., \& Pavlova, M. A. (1990). Changing a natural orientation: Recognition of biological motion pattern by children and adults. Psychologische Beiträge, 32, 28-35.

Neri, P., Morrone, M. C., \& BUrR, D. C. (1998). Seeing biological motion. Nature, 395, 894-896.

Nilsson, L.-G., Olofsson, U., \& NyBerg, L. (1992). Implicit memory of dynamic information. Bulletin of the Psychonomic Society, $\mathbf{3 0}$, 265-267.

Olofsson, U., Nyberg, L., \& Nilsson, L.-G. (1997). Priming and recognition of human motion patterns. Visual Cognition, 4, 373-382.
Pavlova, M. A. (1989). The role of inversion in perception of biological motion pattern [Abstract]. Perception, 18, 510.

Pavlova, M. A. (1992). Object perception through motion in reverse transformation. In G. Borg \& G. Neely (Eds.), Fechner Day '92: Proceedings of the 8th Annual Meeting of the International Society for Psychophysics (pp. 159-164). Stockholm: Stockholm University Press.

PavLova, M. A. (1993). Object recognition through motion in inverted conditions: The effect of a priori information. Perception, 22(Suppl.), 106.

Pavlova, M. A. (1995). Biological motion perception under various presentation rates [Abstract]. Perception, 24(Suppl.), 112.

Pavlova, M., Sokolov, A., \& Bülthoff, I. (1998). Recovery of a priori known structure from biological motion. In B. Bril, A. Ledebt, G. Dietrich, \& A. Roby-Brami (Eds.), Advances in perception-action coupling (pp. 64-68). Paris: Éditions EDK.

Perrett, D. I., Harries, M. H., Benson, P. J., Chitty, A. J., \& Mistlin, A. J. (1990). Retrieval of structure from rigid and biological motion: An analysis of the visual responses of neurons in the macaque temporal cortex. In A. Blake \& T. Troscianko (Eds.), AI and the eye (pp. 181-200). Chichester, U.K.: Wiley.

Proffitt, D. R., Bertenthal, B. I., \& Roberts, R. J., Jr. (1984). The role of occlusion in reducing multistability in moving point-light displays. Perception \& Psychophysics, 36, 315-323.

RUNESON, S. (1994). Perception of biological motion: The KSDprinciple and the implications of a distal versus proximal approach. In G. Jansson, W. Epstein, \& S. S. Bergström (Eds.), Perceiving events and objects (pp. 383-405). Hillsdale, NJ: Erlbaum.

RUNESON, S., \& Frykholm, G. (1983). Kinematic specification of dynamics as information basis for the perception and action perception: Expectation, gender recognition, and deceptive intention. Journal of Experimental Psychology: General, 112, 585-615.

Shiffrar, M., Lichtey, L., \& Heptulla Chatterjee, S. (1997). The perception of biological motion across apertures. Perception \& Psychophysics, 59, 51-59.

SUGiE, N., \& KATo, K. (1987). A computational model for biological motion perception. IEEE Montech Conference on Biomedical Technologies (pp. 140-143). New York: IEEE.

Sumi, S. (1984). Upside-down presentation of the Johansson moving light pattern. Perception, 13, 283-286.

Thornton, I. M., Pinto, J., \& Shiffrar, M. (1998). The visual perception of human locomotion. Cognitive Neuropsychology, 15, 535552 .

Verfaillie, K. (1993). Orientation-dependent priming effects in the perception of biological motion. Journal of Experimental Psychology: Human Perception \& Performance, 19, 992-1013.

WEBB, J. A., \& AGGARWAL, J. K. (1982). Structure from motion of rigid and jointed objects. Artificial Intelligence, 19, 107-130.

(Manuscript received February 16, 1999; revision accepted for publication July 20,1999.) 